\title{
Video Article \\ Determination of Mitochondrial Membrane Potential and Reactive Oxygen Species in Live Rat Cortical Neurons
}

\author{
Dinesh C. Joshi ${ }^{1}$, Joanna C. Bakowska ${ }^{1}$ \\ ${ }^{1}$ Department of Molecular Pharmacology and Experimental Therapeutics, Loyola University Chicago \\ Correspondence to: Joanna C. Bakowska at jbakowska@lumc.edu \\ URL: https://www.jove.com/video/2704 \\ DOI: doi:10.3791/2704
}

Keywords: Neuroscience, Issue 51, Mitochondrial membrane potential, reactive oxygen species, neuroscience, cortical neurons

Date Published: 5/23/2011

Citation: Joshi, D.C., Bakowska, J.C. Determination of Mitochondrial Membrane Potential and Reactive Oxygen Species in Live Rat Cortical Neurons. J. Vis. Exp. (51), e2704, doi:10.3791/2704 (2011).

\section{Abstract}

Mitochondrial membrane potential $\left(\Delta \Psi_{\mathrm{m}}\right)$ is critical for maintaining the physiological function of the respiratory chain to generate ATP. A significant loss of $\Delta \Psi_{\mathrm{m}}$ renders cells depleted of energy with subsequent death. Reactive oxygen species (ROS) are important signaling molecules, but their accumulation in pathological conditions leads to oxidative stress. The two major sources of ROS in cells are environmental toxins and the process of oxidative phosphorylation. Mitochondrial dysfunction and oxidative stress have been implicated in the pathophysiology of many diseases; therefore, the ability to determine $\Delta \Psi_{m}$ and ROS can provide important clues about the physiological status of the cell and the function of the mitochondria.

Several fluorescent probes (Rhodamine 123, TMRM, TMRE, JC-1) can be used to determine $\Delta \psi m$ in a variety of cell types, and many fluorescence indicators (Dihydroethidium, Dihydrorhodamine 123, $\mathrm{H}_{2} \mathrm{DCF}-\mathrm{DA}$ ) can be used to determine ROS. Nearly all of the available fluorescence probes used to assess $\Delta \Psi \mathrm{m}$ or ROS are single-wavelength indicators, which increase or decrease their fluorescence intensity proportional to a stimulus that increases or decreases the levels of $\Delta \Psi \mathrm{m}$ or ROS. Thus, it is imperative to measure the fluorescence intensity of these probes at the baseline level and after the application of a specific stimulus. This allows one to determine the percentage of change in fluorescence intensity between the baseline level and a stimulus. This change in fluorescence intensity reflects the change in relative levels of $\Delta \Psi \mathrm{m}$ or ROS. In this video, we demonstrate how to apply the fluorescence indicator, TMRM, in rat cortical neurons to determine the percentage change in TMRM fluorescence intensity between the baseline level and after applying FCCP, a mitochondrial uncoupler. The lower levels of TMRM fluorescence resulting from FCCP treatment reflect the depolarization of mitochondrial membrane potential. We also show how to apply the fluorescence probe $\mathrm{H}_{2}$ DCF-DA to assess the level of ROS in cortical neurons, first at baseline and then after application of $\mathrm{H}_{2} \mathrm{O}_{2}$. This protocol (with minor modifications) can be also used to determine changes in $\Delta \Psi \mathrm{m}$ and ROS in different cell types and in neurons isolated from other brain regions.

\section{Video Link}

The video component of this article can be found at https://www.jove.com/video/2704/

Protocol

\section{Cell culture}

1. Cortical neurons are isolated and grown using previously described techniques and plated on culture dishes with a glass bottom (MatTek Corporation, Ashland, MA ) coated with poly-D-lysine and laminin ${ }^{1}$.

\section{Preparing the stock solutions for the fluorescent probes TMRM and $\mathrm{H}_{2} \mathrm{DCF}-\mathrm{DA}$}

1. Prepare a 10-mM stock solution of TMRM by dissolving $5.0 \mathrm{mg}$ of TMRM in $1 \mathrm{ml}$ of anhydrous dimethylsulfoxide. Vortex it for $1 \mathrm{~min}$. Then, make aliquots and store them at $-20^{\circ} \mathrm{C}$, protect from light, and use within one month.

2. Next, prepare a 10-mM stock solution of $\mathrm{H}_{2}$ DCF-DA by dissolving $4.87 \mathrm{mg}$ of $\mathrm{H}_{2}$ DCF-DA in $1 \mathrm{ml}$ of anhydrous DMSO. Similarly, vortex it for 1 $\min$. Then, make aliquots and store them at $-20^{\circ} \mathrm{C}$, protect from light, and use within one week.

\section{Loading rat cortical neurons with TMRM and $\mathrm{H}_{2}$ DCF-DA}

TMRM is a potentiometric, cell-permeable fluorescent indicator that accumulates in the highly negatively charged interior of mitochondria. It is important to use the low concentrations (10-50 nM range) of TMRM to avoid auto-quenching of mitochondrial TMRM. Then, the fluorescence signal of TMRM can be directly co-related to $\Delta \Psi_{\mathrm{m}}$ across the inner mitochondrial membrane. A loss of $\Delta \Psi_{\mathrm{m}}$ causes TMRM to leak from mitochondria resulting in a loss of fluorescence intensity. $\mathrm{H}_{2}$ DCF-DA is cell-permeable probe converted into DCF-DA by intracellular esterases, 
and its oxidation results in fluorescent DCF. The final concentration of $\mathrm{H}_{2} \mathrm{DCF}-\mathrm{DA}$ ranges between $2-10 \mu \mathrm{M}$ and it should be tested empirically in neurons derived from different brain regions since high loading concentrations might result in the saturation of DCF fluorescence even in the absence of $\mathrm{H}_{2} \mathrm{O}_{2}$. The presence of any endogenous or exogenous oxidant (e.g., nitric oxide, hydrogen peroxide) will increase DFC fluorescence intensity. Below, we provide a protocol for loading rat cortical neurons with TMRM and $\mathrm{H}_{2}$ DCF-DA.

1. To load the rat cortical neurons with TMRM, first, wash the cultured neurons 3 times with Tyrode's buffer (Text Overlay: TB: $145 \mathrm{mM}$ NaCl, 5 $\mathrm{mM} \mathrm{KCl}, 10 \mathrm{mM}$ glucose, $1.5 \mathrm{mM} \mathrm{CaCl}_{2}, 1 \mathrm{mM} \mathrm{MgCl}_{2}$, and $10 \mathrm{mM} \mathrm{HEPES}$; adjust $\mathrm{pH}$ to 7.4 with $\mathrm{NaOH}$ ). Then, prepare $20 \mathrm{nM}$ of TMRM by diluting the $10 \mathrm{mM}$ TMRM stock 1/1000 times in TB and then add $2 \mu \mathrm{l}$ of diluted TMRM per $1 \mathrm{ml}$ of TB. Incubate the neurons with TMRM for $45 \mathrm{~min}$ in the dark at room temperature. After $45 \mathrm{~min}$, mount the culture dish on the stage of the microscope and start imaging.

2. To load the rat cortical neurons with $\mathrm{H}_{2}$ DCF-DA, wash the cultured neurons 3 times with TB. Next, prepare $2 \mu M$ of $\mathrm{H}_{2} \mathrm{DCF}-\mathrm{DA}$ by diluting the $10 \mathrm{mM} \mathrm{H}_{2}$ DCF-DA stock $1 / 10$ times in TB and then add $2 \mu \mathrm{l}$ of diluted $\mathrm{H}_{2}$ DCF-DA per $1 \mathrm{ml}$ of TB. Then, incubate the neurons with $\mathrm{H}_{2} \mathrm{DCF}-$ DA for $45 \mathrm{~min}$ in dark at room temperature. After $45 \mathrm{~min}$, wash the neurons 4 times with TB to remove excess fluorescent indicator before obtaining images.

\section{Live imaging of neurons incubated with TMRM to determine $\Delta \Psi \mathrm{m}$}

1. To perform live imaging of neurons incubated with TMRM, confocal laser scanning microscopy (Text Overlay: LSM 510, Carl Zeiss Inc.), with the application of live time-series program, is used. Apply low-resolution and attenuated laser power (Text Overlay: low resolution: 256 x 256 ; laser power: $1 \%$ ) to minimize the time needed to obtain images and to avoid photobleaching.

2. . Next, adjust the focus of the mounted neurons loaded with TMRM using reflected light. Examine the TMRM fluorescence by illumination at $514 \mathrm{~nm}$ and detection at $570 \mathrm{~nm}$. Set the detection gain of a camera just below the saturation level.

3. Once all parameters which include resolution, laser power, detection gain of a camera, and time-lapse interval to obtain images are set; do not change these settings between experiments. Next, change the field. Start collecting images.

4. To test changes in $\Delta \Psi \mathrm{m}$, stimuli such as $1 \mu \mathrm{M}$ of FCCP or $2 \mu \mathrm{g} / \mathrm{ml}$ of oligomycin, can be applied, which will significantly depolarize or hyperpolarize the mitochondrial membrane potential, respectively. These changes will be reflected by a decrease in TMRM fluorescence intensity compared with the baseline fluorescence intensity in the case of FCCP, or an increase in TMRM fluorescence intensity in the case of oligomycin.

\section{Live imaging of neurons incubated with $H^{2} D C F-D A$ to determine ROS}

1. To perform live imaging of neurons incubated with $\mathrm{H}_{2} \mathrm{DCF}-\mathrm{DA}$, first, mount the culture dish on the stage of a microscope. Adjust the focus of the cells using reflected light. Examine DCF fluorescence by excitation at $488 \mathrm{~nm}$ and emission at $515 \mathrm{~nm}$.

2. Next, adjust the laser power to $5-7 \%$, detector gain, and resolution of $256 \times 256$. Do not change these settings between experiments. Then, set the frequency for obtaining live images using the time series program.

3. Select a new field and start acquiring images. To detect changes in ROS levels, treat cells with $100-200 \mu \mathrm{M}$ of $\mathrm{H}_{2} \mathrm{O}_{2}$. This will be reflected by an increase in DCF fluorescence intensity compared with baseline level.

\section{Data analysis}

1. Use region of interest (Text Overlay: ROI) tool from the LSM program to select the areas. Then, measure the TMRM or ROS fluorescence intensities. Select ROIs from mitochondrial regions or ROls from the entire cell body in imaged cells to measure the fluorescence intensities of TMRM or ROS, respectively.

2. Calculate the average fluorescence intensities from all ROls of each cell for TMRM or from whole cell bodies for all imaged cells for ROS for each time point. Select regions next to the cells to calculate the background fluorescence intensity. Take several measurements and calculate the average background intensity.

3. Subtract the average background fluorescence intensity from average fluorescence intensities of ROls in each cell for each time point using Microsoft Excel. After subtracting background intensity, normalize the TMRM or DCF fluorescence intensity to the baseline fluorescence using this formula (Text Overlay: $\Delta \mathrm{F}=\mathrm{F}-\mathrm{F}_{\mathrm{o}} / \mathrm{F}_{\mathrm{o}} \times 100$, where $\mathrm{F}=$ fluorescence intensity at any time point, Fo= baseline fluorescence). Then, use the Sigma Plot program to generate the plot showing the changes in fluorescence intensity over time.

\section{Representative Results}

Figure $1 \mathrm{~A}$ shows a fluorescence image of rat cortical neurons incubated with TMRM. Addition of FCCP, a mitochondrial uncoupler, leads to mitochondrial depolarization and a loss of TMRM fluorescence intensity (Fig. 1B). The baseline TMRM fluorescence level remains stable before addition of FCCP (the first $350 \mathrm{sec}$; Fig. 1C). Quantitative analysis of TMRM fluorescence changes over time shows a significant decrease in TMRM fluorescence after addition of FCCP (Fig. 1C).

Figure 1D shows the fluorescence image of rat cortical neurons loaded with DCF. Addition of $\mathrm{H}_{2} \mathrm{O}_{2}$ results in increased DCF fluorescence intensity in cell bodies (Fig. 1E). The baseline DCF fluorescence level is unchanged (the first $120 \mathrm{sec}$ ) before application of $\mathrm{H}_{2} \mathrm{O}_{2}$. Time-lapse measurements of DCF fluorescence show its steady levels, which increase after $\mathrm{H}_{2} \mathrm{O}_{2}$ treatment (Fig. 1F). 

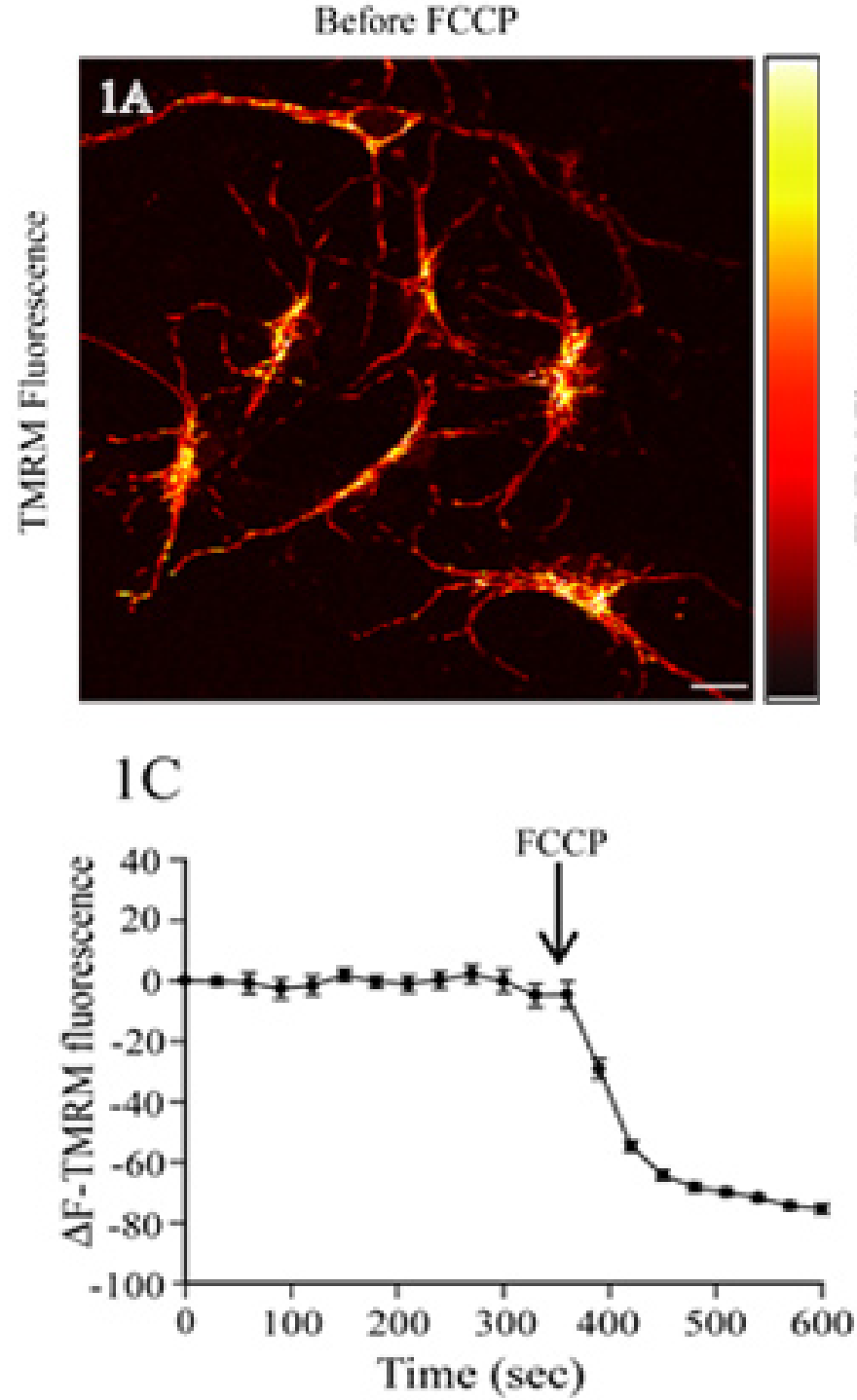

After FCCP

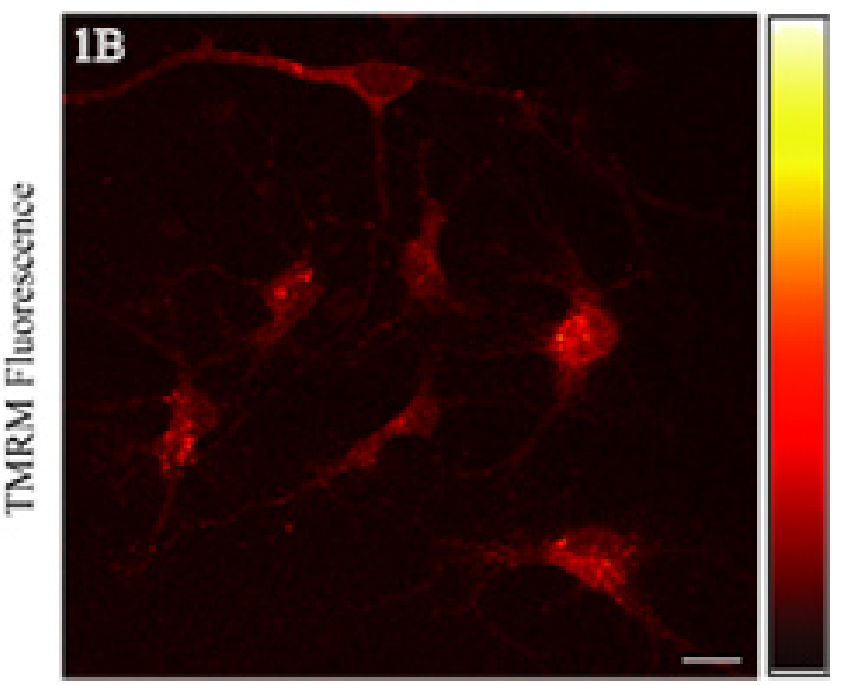

Before $\mathrm{H}_{2} \mathrm{O}_{2}$

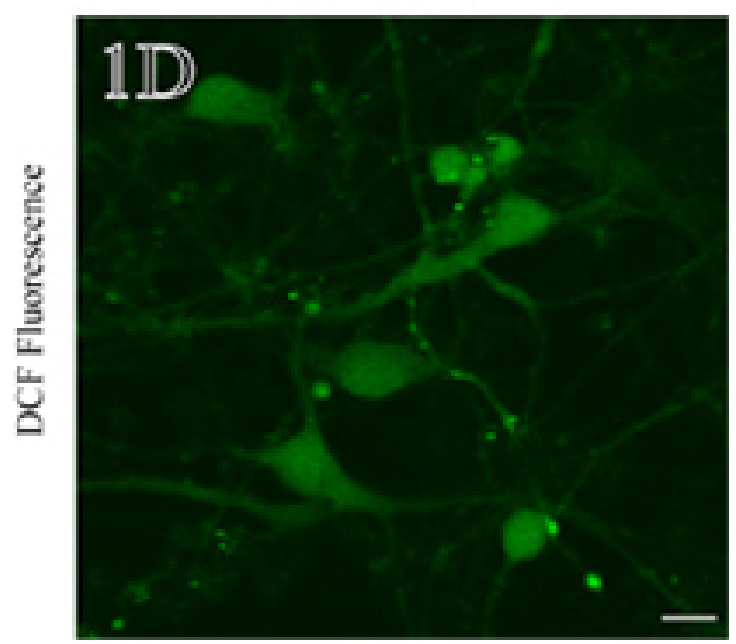

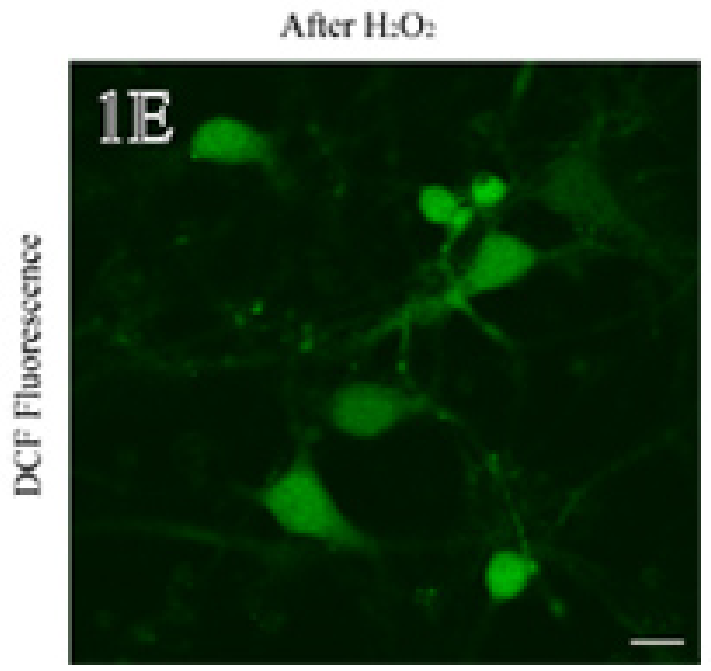

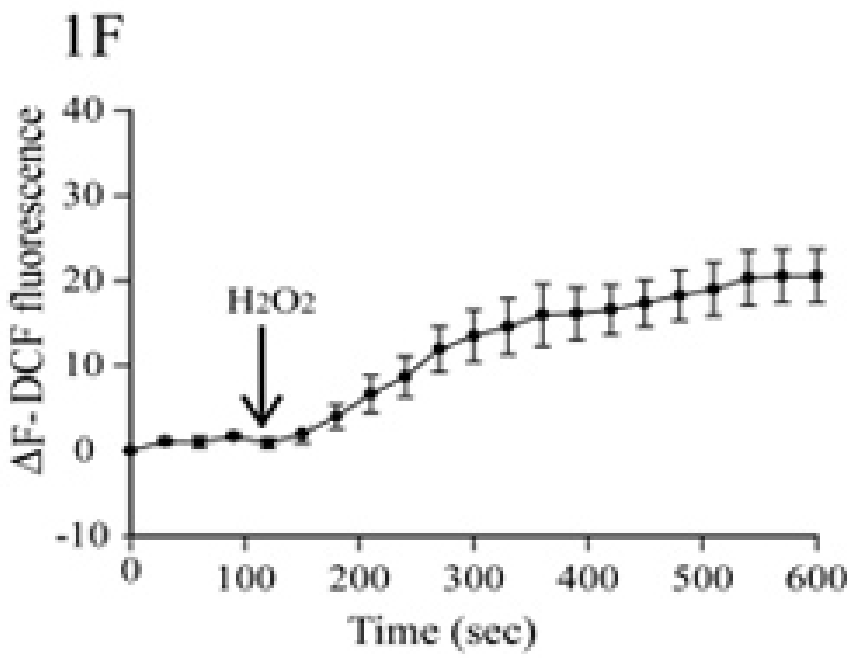

Figure 1. Assessment of mitochondrial membrane potential and ROS levels in live rat cortical neurons. (A) Representative fluorescence image of cortical neurons loaded with TMRM. After scanning the baseline TMRM fluorescence, neurons were treated with the protonophore FCCP $(1 \mu \mathrm{M})$. To the right is a pseudocolor intensity bar of TMRM fluorescence with bright yellow and black representing maximum and minimum 
intensity, respectively. The loss of TMRM fluorescence from the mitochondrial regions indicates the collapse of $\Delta \Psi_{\mathrm{m}}$ upon FCCP treatment (panel B). The quantitative representation of change in TMRM fluorescence intensity at different time points before and after FCCP treatment is shown in panel C. (D) Fluorescence image of rat cortical neurons loaded with H2DCF-DA. After determining the baseline DCF fluorescence, cells were treated with $200 \mu \mathrm{M} \mathrm{H}_{2} \mathrm{O}_{2}$, and the change in DCF fluorescence was assessed. An increase in the DCF fluorescence reflects the increase in ROS levels upon $\mathrm{H}_{2} \mathrm{O}_{2}$ treatment $(E)$. Quantitative analysis of change in DCF fluorescence, before and after $\mathrm{H}_{2} \mathrm{O}_{2}$ treatment, is shown in panel F. Scale bar $=10 \mu \mathrm{m}$

\section{Video.7.1 - labmedia 2704_Joshi.avi}

Live cell imaging of TMRM in cortical neurons before and after FCCP addition using 40X objective. The pseudocolor intensity shows a maximum (bright yellow, before FCCP addition) and decreased (red color, after FCCP addition) TMRM fluorescence intensity after FCCP addition. Click here to view video

Video. 7.5 - labmedia 2704_Joshi.avi

Live cell imaging of DCF in cortical neurons before and after $\mathrm{H}_{2} \mathrm{O}_{2}$ addition using $40 \mathrm{X}$ objective. The baseline DCF fluorescence has light green color in cell bodies and $\mathrm{H} 2 \mathrm{O} 2$ addition increases the DCF fluorescence intensity to bright green color. Click here to view video

\section{Discussion}

We have presented a step-by-step procedure describing how to determine $\Delta \Psi_{\mathrm{m}}$ and ROS in rat cortical neurons using the fluorescent indicators TMRM and $\mathrm{H}_{2}$ DCF-DA, respectively. For other cell types, it is important to empirically determine the final concentration and loading time for TMRM or $\mathrm{H}_{2}$ DCF-DA. In general, TMRM concentrations range from 20-200 nM, and the cell incubation time with TMRM varies from 20 to 60 min. The final concentration of $\mathrm{H}_{2}$ DCF-DA ranges from 2-10 $\mu \mathrm{M}$, and incubation of cells in a loading solution containing this indicator varies from $30-45 \mathrm{~min}$.

It is important to optimize the laser power and scan speed of taking the images to avoid both photo-toxicity to the cells and changes in the fluorescence intensity (for example flickering of TMRM fluorescence) in the absence of any stimulus. The optimized optical settings should result in a fluorescence signal that is not over or under saturated (threshold) in the absence of stimulus. The optimal conditions to collect the images from a selected field at a particular laser power and a scan speed are achieved when there are no changes in the fluorescence intensity of the probe in the absence of any stimulus for $10-15$ min of live imaging

Other fluorescence probes to determine $\Delta \Psi \mathrm{m}$ include rhodamine 123 and tetra methyl rhodamine ethyl ester (TMRE). However, they were found to inhibit the respiratory processes in isolated mitochondria ${ }^{2}$. Importantly, TMRM has no effect on mitochondrial respiration at low concentrations ${ }^{2}$ and has low phototoxicity and photobleaching ${ }^{3}$ compared with other probes. $\mathrm{H}_{2}$ DCF-DA is a good indicator for ROS as it is well retained in the cells and recognizes several oxidant species, such as peroxides, super oxides, and nitric oxide ${ }^{4}$.

\section{Disclosures}

No conflicts of interest declared.

\section{Acknowledgements}

This work was supported by the National Institutes of Health (K22NS050137 to J.C.B.).

\section{References}

1. Hilgenberg, L. G. \& Smith, M. A. Preparation of dissociated mouse cortical neuron cultures. J. Vis. Exp. 10,562 (2007)

2. . Scaduto, R. C.,Jr \& Grotyohann, L. W. Measurement of mitochondrial membrane potential using fluorescent rhodamine derivatives. Biophys. J. 76, 469-477 (1999).

3. Ward, M. W. et al. The amyloid precursor protein intracellular domain (AICD) disrupts actin dynamics and mitochondrial bioenergetics. J. Neurochem. 113, 275-284 (2010).

4. Gunasekar, P. G., Kanthasamy, A. G., Borowitz, J. L. \& Isom, G. E. NMDA receptor activation produces concurrent generation of nitric oxide and reactive oxygen species: implication for cell death. J. Neurochem. 65, 2016-2021 (1995). 